\title{
A Study of Waste Water Treatment of Microbiological Laboratories of Hospitals by Electrolyzed Oxidized Water
}

\author{
Fiza Sarwar \\ College of Earth \& Environmental Sciences \\ University of the Punjab, Lahore, Pakistan \\ Tel: 92-0454-710-958_E-mail: fiza_sarwar@yahoo.com \\ Aroos Munir (Corresponding author) \\ College of Earth \& Environmental Sciences \\ University of the Punjab, Lahore, Pakistan \\ Tel: 92-042-682-2812 E-mail: aroosmunir@yahoo.com \\ Ilyas Ahmed Faridi \\ Institute of Public Health, 6 Birdwood Road Lahore, Pakistan \\ Tel: 300-414-1850 E-mail: haq_farid@yahoo.com
}

Received: August 18, 2010 Accepted: October 12, 2010 doi:10.5539/gjhs.v3n1p150

\begin{abstract}
Hospital liquid infectious waste is one of the most important aspects of water contamination. The present investigation was undertaken to evolve a cost effective alternate method of waste water treatment by using Oxidized Water as a disinfectant for hospital effluents. Liquid infectious waste coming from diagnostic laboratories of hospitals (Urine, Blood and Mix of both) was treated with electrolyzed Oxidized Water. Different v/v ratios $(95: 5,85: 15,75: 25,50: 50$ and 25:75) of Sample to Electrolyzed Oxidized Water (EOW) were cultured and incubated at $37^{\circ} \mathrm{C}$ for 24 hours. EOW showed a direct relationship with the decontamination of inorganic urine but for blood, an unidentified pattern was observed that may be due to change in $\mathrm{pH}$ and/or Oxidation Reduction Potential (ORP) of EOW because of organic nature of blood. The most effective ratio of sample to EOW, at which decontamination was found to be maximum for urine, was 25:75 with treatment efficiency of $96.15 \%$ and $85: 15$ with treatment efficiency of $84.8 \%$ for blood. The study revealed that Electrolyzed Oxidized Water may be used as a better alternative for treatment of liquid infectious waste.
\end{abstract}

Keywords: Treatment, Microbiological, Hospital, Electrolyzed, Oxidized and water

Abbreviations: EOW: Electrolyzed Oxidized Water, ORP: Oxidation Reduction Potential

\section{Introduction}

One of the burning issues of environmental pollution is water contamination due to direct discharge of hospital liquid infectious waste into sewage. Blood and urine are the major infectious effluents from diagnostic laboratories, which require treatment prior to disposal into sewage. The methods currently being used for treatment of infectious waste are either expensive, require large space and man power for its continuous sustainability or become source of pollution themselves. For instance, incineration result in emission of carcinogenic compounds like dioxins, waste water treatment plant requires huge space while, chemical disinfection by chlorine, ammonium salts and phenolic compounds (Pruss et al, 1999) may lead to secondary pollution.

EOW is generated by electrolysis of a dilute aqueous solution of sodium chloride $(\mathrm{NaCl})$ in an electrolysis chamber where the anode (+ electrode) and cathode (- electrode) are separated by a cationic membrane to form two compartments (Kumon, 1997). The anode acidic EOW has a low $\mathrm{pH}$ of 2.3 to 3.0, a high oxidation-reduction potential (ORP) greater than $1000 \mathrm{mV}$, and contains relative concentrations of chlorine (Cl2), 
hypochlorous acid (HOCl) and hypochlorite (Sharma and Demirci, 2003). The alkaline solution from the cathode has a high $\mathrm{pH}$ and low ORP (Kumon, 1997).

Electrolyzed oxidizing water (EOW) has proved to be an effective disinfectant, due to its strong microbicidal activities on a wide variety of bacterial pathogens including mycobacteria as well as bacterial endospores (Tanaka et al., 1996; Selkon et al., 1999; Loshon et al., 2001). It is reported that EOW has a potential to inactivate bacterial endotoxins or fungal toxins by conversion of hazardous substances into non harmful components (Suzuki et al., $2002 \mathrm{a}, \mathrm{b}$ ). Large amount of $\mathrm{HOCl}$ at a low $\mathrm{pH}$ of $\sim 3$ enhances the disinfecting efficacy of EOW (Vorobjeva et al., 2004; White, 1999). EOW is found to be a useful disinfectant for hospital infections. They investigated EOW against 10 of the most common opportunistic pathogens which became completely inactive after treatment (Vorobjeva et al., 2004).

The present investigation was undertaken to select the best concentration of EOW to decontaminate the infectious effluents from microbiological laboratory of hospital. Comparison of number of microbial colonies grown in the sample control and treated samples was taken as criteria to determine the best ratio of EOW to treat for maximum decontamination.

\section{Material and methods}

\subsection{Materials}

\subsubsection{Apparatus}

Test tubes, Petri Dishes, Micro Syringes (1 c.c.), Cotton, Conical Flask, Test Tube Stand, Incubator, Laminar Flow Cabinet, Autoclave and Colony Counter.

\subsubsection{Chemicals}

Dermo Zell Electrolyzed Oxidized Water (Bio-Zell+ Pakistan), Nutrient Agar (Lab M. Ltd), Distilled Water

\subsection{Methods}

Liquid infectious waste (Urine, Blood and Mixed Waste) was collected from microbiology laboratory of hospital and different $\mathrm{v} / \mathrm{v}$ ratios of sample to EOW $(95: 5,85: 15,75: 25,50: 50$ and 25:75) were applied on prepared Petri dishes of Nutrient Agar by Spread Plate Technique and labeled. Pure samples of blood urine and mixed waste were also cultured to determine the extent of pollution in terms of number of microbial colonies. Control of EOW was also kept. All the Petri dishes were kept in incubator at $37^{\circ} \mathrm{C}$ and kept for 24 hours. After $24 \mathrm{hrs}$ of incubation, microbial growth in each of the plate was examined. Using Colony Counter, number of colonies in each of the Petri dish was counted to determine the most effective concentration of EOW for decontamination. The treatment efficiency of EOW was determined by applying following formula,

Treatment Efficiency $=$ Ei - Eo $/$ Ei x 100

$\mathrm{Ei}=$ Number of Colonies in Pure sample (Urine/Blood/Mixed sample)

Eo $=$ Number of colonies in treated samples (Urine/Blood/Mixed sample)

\section{Results}

\subsection{Decontamination of Urine}

The experiment revealed that there is a directly proportional relationship between the amount of EOW and reduction in number of microbial colonies is shown in graph 1. The different ratios of urine to EOW i.e. 95:5, $85: 15,75: 25,50: 50$ and 25:75 showed average treatment efficiencies as, 5.59, 15.54, 34.5, 61.7 and 96.15 respectively. The best sample to EOW ratio for treatment of urine is 25:75 with average treatment efficiency of $96.15 \%$. Comparison of microbial growth in control of urine and microbial growth after treatment of urine with $75 \%$ of EOW is shown in figure 1.

\subsection{Decontamination of Blood}

Unlike urine, blood treatment with EOW showed an unidentified pattern of decontamination i.e., reduction in number of colonies was observed at sample to EOW ratios, 95:5 and 85:15 is shown in graph 1. But after 85:15, increase in number of colonies was observed at higher percentages of EOW. The different ratios of Blood samples to EOW i.e. 95:5, 85:15, 75:25, 50:50 and 25:75 showed average treatment efficiencies as, 46.73, 84.81, $75.19,-232.6$ and -47.8 respectively. However, the most effective ratio of blood sample to EOW was found to be $85: 15$ at which the average treatment efficiency calculated as $84.81 \%$. Comparison of microbial growth in control of blood to microbial growth after treatment of blood with $15 \%$ of EOW is shown in figure 2 . 


\subsection{Decontamination of Mixed Waste (Urine and Blood)}

For mixed waste (blood and urine) the effect of EOW with the selected concentrations showed an unidentified pattern is shown in graph 1 . The different ratios of mixed waste sample to EOW i.e. 95:5, 85:15, 75:25, 50:50 and 25:75 showed average treatment efficiencies as, $-167.4,47.69,33.1,67.65$ and 49.72 respectively.

\section{Discussion}

In hospitals for medical purposes such as diagnostics and research a large variety of substances are in use. After application, diagnostic agents, contaminated with viruses, protozoa, and other microorganisms reach the local sanitary sewage system. The bacteria contamination is the most serious risk associated with domestic sewage exfiltration with sufficient virulence to transmit disease. It was needed to find out most cheap and feasible method of liquid infectious waste management. Therefore, Electrolyzed Oxidized water was taken for decontamination of infectious waste prior to discharge into sewage. It was found to be a successful study giving an effective approach in terms of environmental conservation and being socially and economically feasible.

The pattern of growth of microbes shows that ratio of EOW to sample i.e. 75:25, is the best ratio for treatment of urine, with average treatment efficiency of $96.15 \%$. The experiment revealed that there is a directly proportional relationship between the amount of EOW and reduction in the growth of microbial colonies. This is because urine, being inorganic and water based in nature, did not affect $\mathrm{pH}$ of EOW. However, the most effective ratio of EOW to blood sample was found to be $85: 15$ at which the average treatment efficiency calculated as $84.81 \%$. The unidentified pattern shows that the organic nature of blood affects the $\mathrm{pH}$ and/or ORP of EOW from 2.5 towards neutral $\mathrm{pH}$. Secondly some kinds of microbes may activate with change in $\mathrm{pH}$ due to change in concentration of EOW.

The effect of EOW with the selected concentrations showed an unidentified pattern for mixed waste. It is may be due to effect of its organic nature on the $\mathrm{pH}$ and/or ORP of EOW from 2.5 towards neutral $\mathrm{pH}$. Secondly some kinds of microbes may activate with change in $\mathrm{pH}$ due to change in concentration of EOW. Each of the samples showed a different effective percentage of EOW for its decontamination but in each sample effective percentage of EOW for Mixed waste sample matched with effective percentage for blood or urine of the same sample. Having an unidentified pattern it followed the pattern of decontamination of that sample viz. less infectious rather than to follow the pattern of infectious waste sample.

\section{Conclusion}

Use of EOW for decontamination of liquid infectious waste may help overcome the problems associated with other treatment methods. It is most practicable, efficient and user-friendly for all types of heath care facilities. It is also an economic method, without need of costly equipment and continuous supervision. Waste is decontaminated without generation of secondary pollution. To ensure this Blood and Urine, being different in their chemical composition and nature, should be treated with different concentrations of EOW separately prior to disposal.

\section{References}

Kumon, K. (1997). What is functional water? Artif. Organs, 21, pp. 2-4.

Loshon, C. A., Melly, E., Setlow, B., and Setlow, P. (2001). Analysis of the killing of spores of Bacillus subtilis by a new disinfectant, Sterilox ${ }^{\circledR}$. Journal of Applied Microbiology. (91), 1051-1058.

Pruss, A., Girouit E., and Rushbrook, P. (1999). Safe management of waste from health care activities, Geneva, WHO.

Pruss, A., Girouit E., and Rushbrook, P. (2002). Safe management of waste from health care activities, Geneva, WHO, (2), 2-3.

Selkon, J. B., Babb, J. R., and Morris, R. (1999). Evaluation of the antimicrobial activity of new super-oxidized water, Sterilox ${ }^{\circledR}$, for the disinfection of endoscopes. Journal of Hospital Infection. (41), 59-70.

Sharma, R. R., and Demirci, A. (2003). Treatment of Escherichia coli O157:H7 inoculated alfalfa seeds and sprouts with electrolyzed oxidizing water. Int. J. Food Microbiol, 86, pp. 231-237.

Suzuki, T., Itakura, J., Watanabe, M., Ohta, M., Sato, Y., and Yamaya, Y. (2002a). Inactivation of staphylococcal enterotoxin A with an electrolyzed anodic solution. Journal of Agricultural Food and Chemistry, (50), 230-234. 
Suzuki, T., Noro, T., Kawamura, Y., Fukunaga, K., Watanabe, M., Ohta, M., Sugiue, H., Sato, Y., Kohno, M., and Hotta, K. (2002b). Decontamination of Aflatoxin-forming fungus and elimination of Aflatoxin mutagenicity with electrolyzed $\mathrm{NaCl}$ anode solution. Journal of Agricultural Food and Chemistry, (50), 633-641.

Tanaka, H., Hirakata, Y., Kaku, M., Yoshida, R., Takemura, H., Mizukane, R., Ishida, K., Tomoro, K., Koga, H., Kohno, S., and Kamihira, S. (1996). Antimicrobial activity of superoxidzed water. Journal of Hospital Infection, (34), 43-49.

Vorobjeva, N. V., Vorobjeva, L. I., and Kodjaev, E. Y. (2004). The bactericidal effect of electrolyzed oxidizing water on bacterial strains involved in hospital infections. Artificial Organs, (28), 590-592.

White, G. C. (1999). Handbook of chlorination and alternative disinfectants. John Wiley \& Sons, Inc., USA.

Table 1. Decontamination Efficiency of EOW

\begin{tabular}{|l|l|l|l|l|}
\hline \multirow{2}{*}{ Sr No } & \multirow{2}{*}{ Ratio of Sample to EOW } & \multicolumn{3}{|c|}{ Average Treatment Efficiency \% } \\
\cline { 3 - 5 } & & Urine & Blood & $\begin{array}{l}\text { Mixed Waste } \\
\text { (Urine and Blood) }\end{array}$ \\
\hline 1 & $95: 5$ & 5.59 & 46.73 & -167.4 \\
\hline 2 & $85: 15$ & 15.94 & 84.81 & 47.69 \\
\hline 3 & $75: 25$ & 34.5 & 75.19 & 33.1 \\
\hline 4 & $50: 50$ & 61.7 & -232.6 & 67.65 \\
\hline 5 & $25: 75$ & 96.15 & -47.8 & 49.72 \\
\hline
\end{tabular}

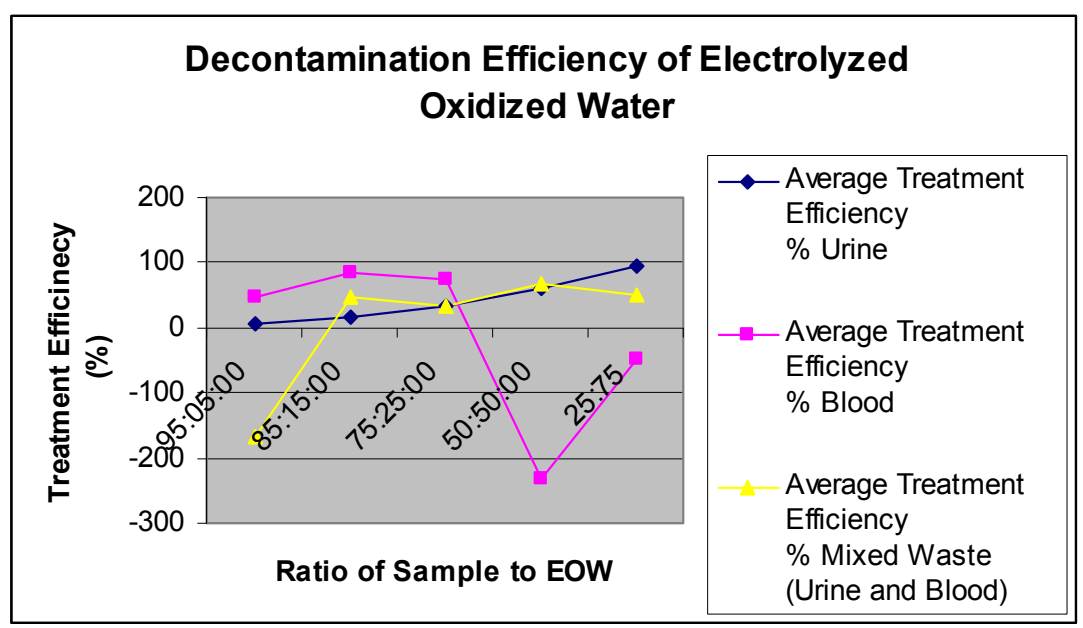

Graph 1. Decontamination efficiency of Electrolyzed Oxidized Water (EOW) 


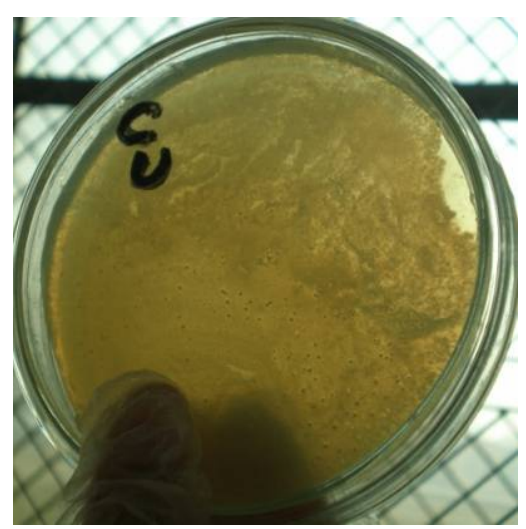

(a)

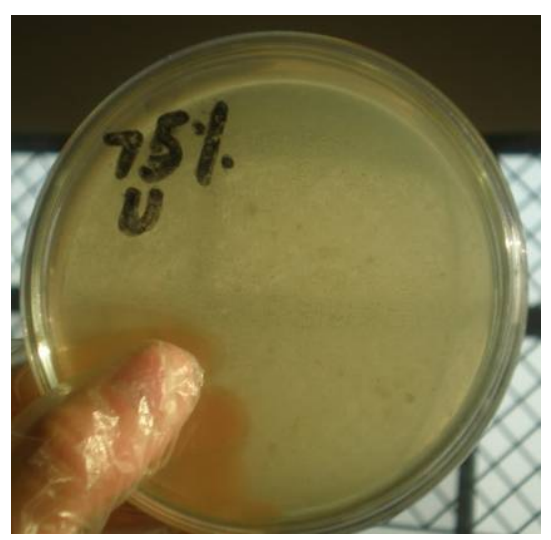

(b)

Figure 1. Comparison of Microbial growth in control of Urine (a) and Microbial growth after treatment of Urine with $75 \%$ of EOW (b)

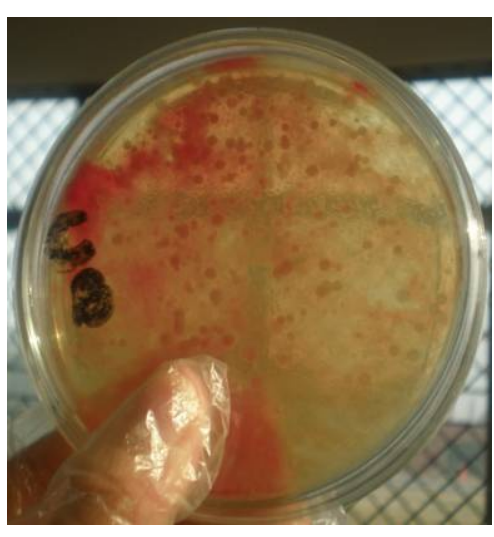

(a)

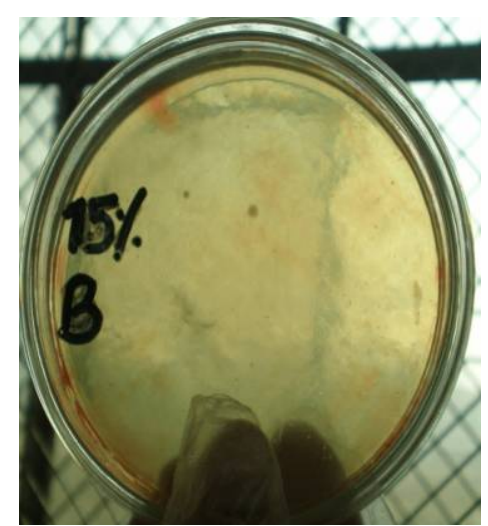

(b)

Figure 2. Comparison of Microbial growth in control of Blood(a) to Microbial growth after treatment of blood with $15 \%$ of EOW (b)

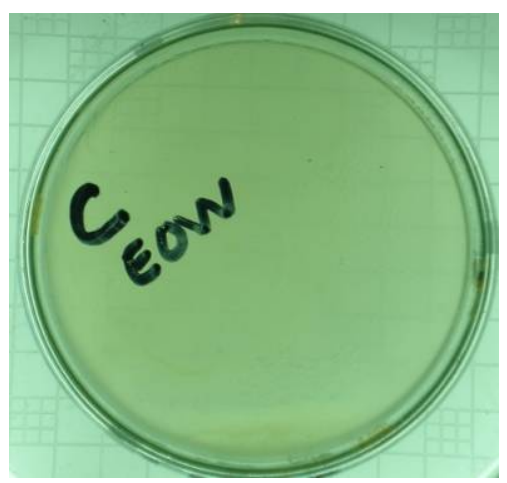

Figure 3. Control of EOW showing no microbial growth 